\title{
The Study of П-Ctalytic Cycles in Inertial Confinement Fusion D/T in Dense Plasma
}

\author{
M. Mahdavi ${ }^{1, *}$, N. Ghal-Eh ${ }^{2}$, Gh. Aghasian ${ }^{2}$ \\ ${ }^{1}$ Physics Department, University of Mazandaran, P. O. Box 47415-416, Babolsar, Iran \\ ${ }^{2}$ School of Physics, Damghan University, Damghan, Iran \\ *Corresponding Author: m.mahdavi@umz.ac.ir
}

Copyright (C 2014Horizon Research Publishing All rights reserved

\begin{abstract}
There will be the possibility of producing Pion particles in the collision of relativistic electrons and fuel pellet ions in the fast ignition. The produced Pions, in collisions with nuclei of hydrogen atoms (ions of D/T plasma) get slow, will eventually lead to the production of Pion atoms. The produced Pion atoms, in a very short time, will create Pion molecules. During this process, because of reduction in the Coulomb repulsion between the nuclei of fuel by Pion, the possibility of nuclear fusion between isotopes $\mathrm{D} / \mathrm{T}$ will be provided. Dynamical equations governing the particle density of the cycle have been resolved in optimal concentration by Monte Carlo method and LSOD code. The obtained results show that although the relative density of Pion, reaches its minimum amount after a limited time about $40 \mathrm{~ns}$, but the fusion process of $\pi \mathrm{dt}$ Pion molecules will occur within $10 \mathrm{~ns}$. According to the short life time of Pion particles, it is observed that the number of cycles which Pion catalyzes in its life time is not enough to produce the optimization energy.
\end{abstract}

Keywords Pion Catalysis, Relativistic Electron, Dense Plasma, Inertial Confinement Fusion

PACS Number: 25.80.Ls; 25.60Pj; 28.52.-s

\section{Introduction}

One of the methods of producing nuclear fusion energy is ICF, which is taken place in three ways of direct ignition, indirect ignition, and fast ignition. In all of these methods, because very different physical conditions with surrounding environment for the fuel, the fuel is unstable [1], so, the fusion conditions will be available for a short time. Thus, the problems directed the scientists to other methods of fusion called cold fusion. One way to do fusion reaction at relatively low temperature is to use particles like Mion, Pion, etc. as a catalyst.

In this method, the process of fusion is done based on making Coulomb repulsion forces and Coulomb barrier between nuclei ineffective or less effective and bringing them closer together. In this approach, in contrast to (ICF) and (MCF) methods, fusion is possible at room temperature and not high densities. Therefore, the particles should be used to replace the atomic electrons, and its Bohr orbit radius must be smaller than the radius of Bohr orbit of the hydrogen atom so that the target nucleus can be discharged and reduces the distance of its nucleus with other fusion nucleus and increase the possibility of fusion. Because the new particle must replace the electron, it should have negative charge. On the other hand, because after the entrance of catalyst particle, which is heavier than the electron, the Bohr radius of a new atom is reduced as $\mathrm{a}_{0}=\frac{\hbar}{\mathrm{mc} \alpha}$, to reduce Bohr radius the mass of the particle must be greater than the electron mass, also the proposed particle must have an almost high half-life to reduce the height of Coulomb barrier between the nuclei fusion.

For example, if the catalyst particle is Pion, after a time it slows down and is conquered by the neighborhood atoms and, by giving a part of its energy to the atomic electron of K-orbit, throws the electron out and replaces it. Pionic atoms in the environment collide with other deuterium and tritium nuclei in the environment and make unstable molecules of $\pi \mathrm{dt}$. As a result, the fusion is performed and $17.6 \mathrm{MeV}$ energy is produced in this process. In fact, it is the catalyst particle (Pion) which makes Coulomb barrier ineffective and fusion takes place at very low temperatures, even at room temperature.

\section{Relativistic Electrons in Fast Combustion Process}

Near the point of compression, a small area of fuel, with a radius of about $20 \mu \mathrm{m}$, with an external energy source, is heated. These foreign sources of energy can be from the high energy electron beams that are accelerated by density fuel [2] The electrons are accelerated by a high energy Petawatt laser from the ablated plasma that surrounds the capsule, or from the tip of a cone that is embedded in the capsule [3]. Fig. (1) shows a scheme in which laser creates a channel between the low-density plasma and Petawatt laser pulses, after spreading through channel, remove the critical density of 
plasma and transfer its energy to surface electron. In the design of the cone, the cone wall keeps the plasma, in which the Petawatt pulse lasers can spread. The rapidly distributed electrons to the fuel lose their energy density by hitting to the field electrons and surrounding ions [3].

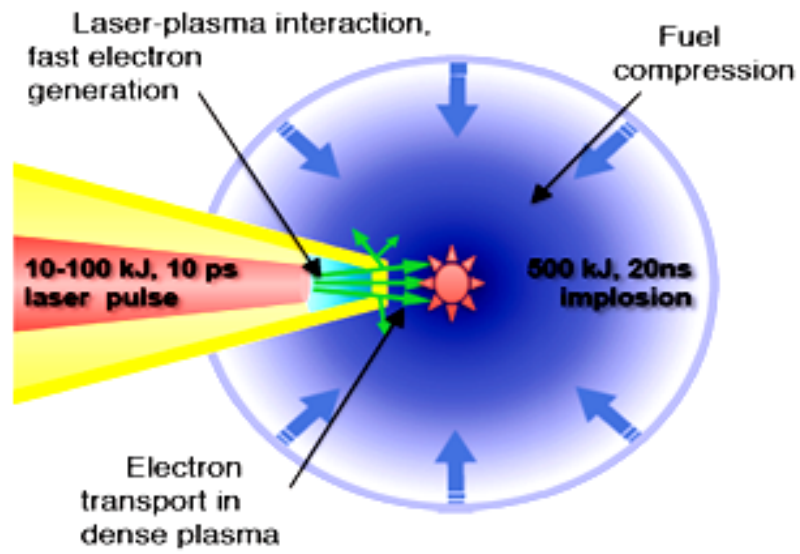

Figure 1. Fast ignition of fuel tablets by powerful lasers

According to the triple process, the collisions of fast electrons with a target ion with $\mathrm{Z}$ charge create the pair electron-positron. Pair production of electron-positron can be directly produced by interaction of electrons with the Coulomb medians of nucleus atoms, or they can be produced indirectly. The reaction rate of pair electron-positron is from $\mathrm{z}^{2} \mathrm{n}_{\text {hot }} \mathrm{n}_{\mathrm{i}} \alpha^{2}$ for direct production in core while it is from $\mathrm{z}^{2} \mathrm{n}_{\text {hot }} \mathrm{n}_{\mathrm{i}} \alpha$ in the indirect method and the reaction rate of production photon pair is from $\mathrm{z}^{2} \mathrm{n}_{\gamma} \mathrm{n}_{\mathrm{i}} \alpha$. In these relations $\alpha=\frac{1}{137}, \mathrm{z}$ is the atomic number, $\mathrm{n}_{\text {hot }} \mathrm{n}_{\mathrm{i}} 6 \mathrm{n}_{\mathrm{e}}, \mathrm{n}_{\gamma}$ are the atomic thermal electron density, atomic electron and photo, respectively [5].

Fast electrons entered in the deuterium-tritium plasma, can cause electrical disintegration. In Figures (2) and (3), the Fynman's graph shows the exchange photons. The first stage of electrical disintegration includes protons getting out of the deuterium and tritium. But the condition for Pion generation will be provided when the energy of fast electron radiation gets to $E_{b} \geq 145 \mathrm{MeV}$. [6]

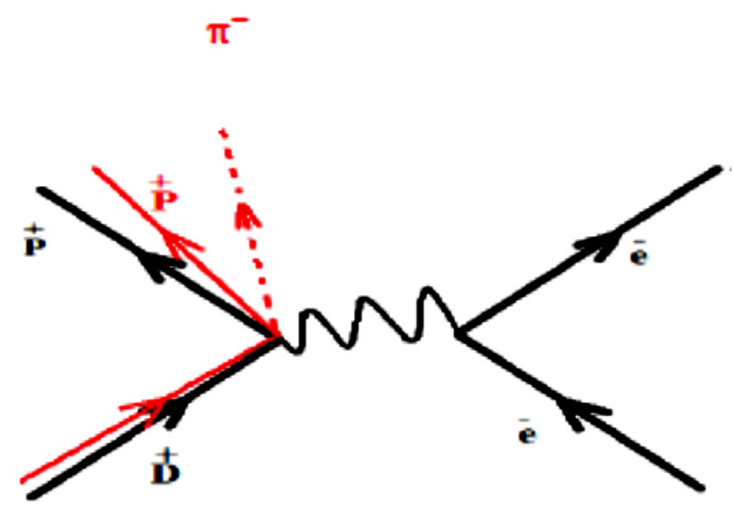

Figure 2. Fynman diagram of the fast electrons collision with $\mathbf{D}^{+}$plasma ions and electro-disintegration

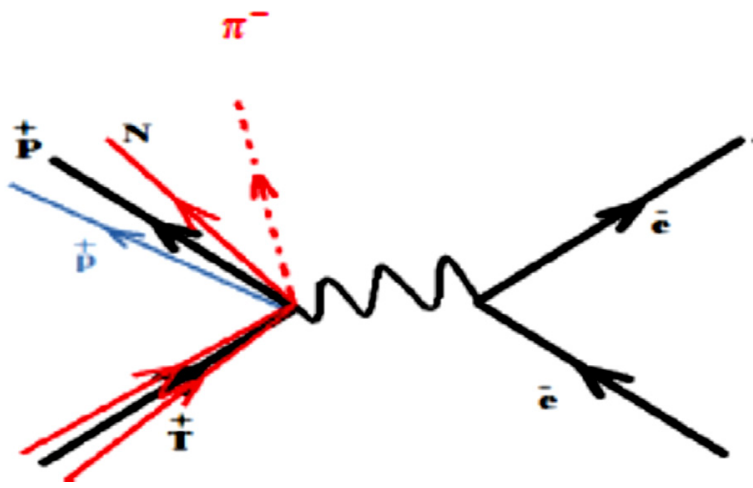

Figure 3. Feynman diagram of the fast electrons collision with $\mathbf{T}^{+}$plasma ions and electro- disintegration

For example, the probability of Pion generation in $5 \mathrm{MeV}$ energy in gold is calculated as follows [7].

$$
\begin{gathered}
\frac{\sigma_{\mathrm{T}}}{\sigma_{\gamma \rightarrow \mathrm{e}^{+} \mathrm{e}^{-}}}= \\
\frac{\alpha}{\pi}\left[\ln \left(\frac{\mathrm{E}}{\mathrm{m}_{\mathrm{e}} \mathrm{c}^{2}}\right) \ln \left(\frac{\mathrm{E}}{2.137 m_{e} \mathrm{c}^{2} \mathrm{Z}^{\frac{-1}{3}}}\right)+\frac{1}{3} \ln ^{2}\left(2.137 Z^{\frac{-1}{3}}\right)\right]=0 / 01
\end{gathered}
$$

in which:

$$
\sigma_{\mathrm{T}}=\left(\frac{28}{27 \pi}\right) \cdot\left(\frac{\mathrm{Zr}_{0}}{137}\right)^{2}\left(\ln \gamma_{\mathrm{b}}\right)^{3}
$$

$\sigma_{\mathrm{T}}$ is the cross section of the three processes. Thus, the possibility of negative Pion generation exists in the fast ignition process due to the collision of the relativistic electrons with the charged particles of fuel. Figure (4) shows the total cross-section diagram of the entire three processes based on $\gamma_{b}=\frac{E_{b}}{m_{e} c^{2}}$.

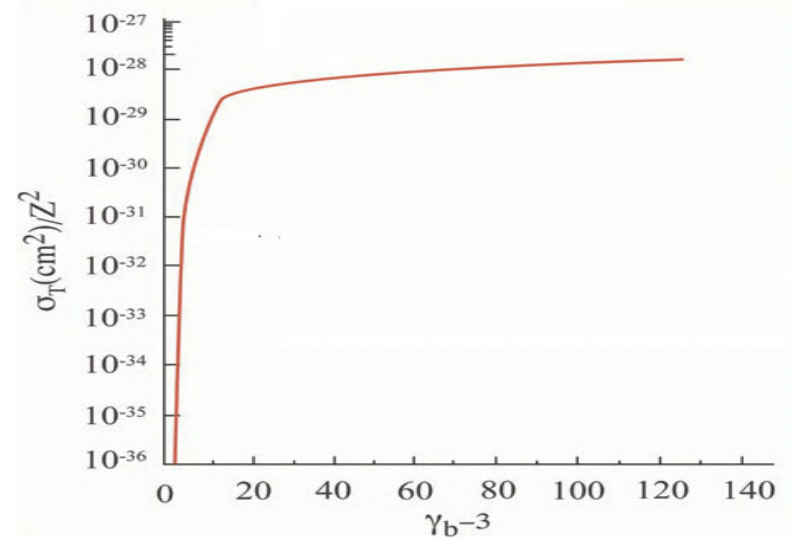

Figure 4. Total cross-section versuse $\boldsymbol{\gamma}_{\mathbf{b}^{-3}}$.

\section{Pion Catalyst Cycle}

According to the arguments raised in the ICF process, the possibility of Pion particles production exists in the interaction of electrons with plasma ions. So, the produced Pion in the hot and dense plasma gives its energy to the plasma by the processes of slowing down. This Pions can catalyzed different cycles for fusion reactions of DD, TT, 
and DT. Also Pion, in its lifetime,( i.e. $28 \mathrm{~ns}$ ), can be completely available in during the confinement time of the target. When heavier negative particles than electrons, such as Pion, are injected into the material, they replace the atomic electrons while slowing down; these atoms sits, because of their high mass, are firstly formed in excited state, and during some processes transit to lower states of energy. If the pion particle catalyst is trapped by hydrogen isotopes, by comparing the binding energy of Pion the final state and electron energy bound which has replaced it, one will have:

$$
E_{n}=\frac{-m c^{2} \alpha^{2}}{2 n^{2}}
$$

and by equaling the energy of two levels in two systems of Pion and electron, it will be:

$$
\frac{E_{n}}{n_{e}}=\sqrt{\frac{m_{e}\left(m_{p}+m_{\pi}\right)}{m_{\pi}\left(m_{p}+m_{e}\right)}}
$$

In eq. (4), $\mathrm{m}_{\mathrm{p}}$ is proton mass, $\mathrm{m}_{\pi}$ Pion mass, and $\mathrm{m}_{\mathrm{e}}$ is electron mass; also, $\frac{\mathrm{m}_{\mathrm{e}}}{\mathrm{m}_{\pi}} \cong 273$, and by selecting $\mathrm{n}_{\mathrm{e}}=1$ for hydrogen isotopes, the number of level which the Pion particle has been trapped in hydrogen atom and made the Pion atom, is calculated as follows:

$$
\mathrm{n}_{\pi} \approx \sqrt{\frac{\mathrm{m}_{\mathrm{e}}}{\mathrm{m}_{\pi}}} \approx 16
$$

so, the state of Pion in the first entry will be in $n_{\pi}=16$. Because the formed Pion atom is in excited state, the Pion, as a cascade, transits to lower-energy states to reach the ground state of $1 \mathrm{~S}$.

According to Fig. 5, Pion particle, passes a cycle after entering the plasma fuel. This particle, after entering into the plasma fuel, immediately slowing down and produces atoms $\pi \mathrm{d}$ or $\pi \mathrm{t}$ with $\lambda_{\mathrm{a}}$ rate. $\pi \mathrm{d}$ atoms can form $\pi \mathrm{dd}$ molecules with the rate of $\lambda_{\pi d d}$ or under isotopic transfer with the rate of $\lambda_{\mathrm{dt}}$ changes into atoms. The $\pi \mathrm{t}$ produced atoms can form $\pi d t$ Pion molecules with the rate of $\lambda_{\pi d t}$, and $\pi \mathrm{tt}$ with the rate of $\lambda_{\pi t t}$. The produced Pion molecules, in certain circumstances, do the fusion process and Pions, with the probability of $\left(1-\mathrm{w}_{(}\right)$set free or with the probability of $\mathrm{w}_{()}$stick to fusion products. In all of these reactions, the released Pion gets back into the cycle and catalyzes another fusion process. However, if Pion sticks to the $\alpha$ particle, Pion will get out of the cycle or with the rate of $\lambda^{\mathrm{f}}$ will decay and it cannot process catalyze the fusion process.

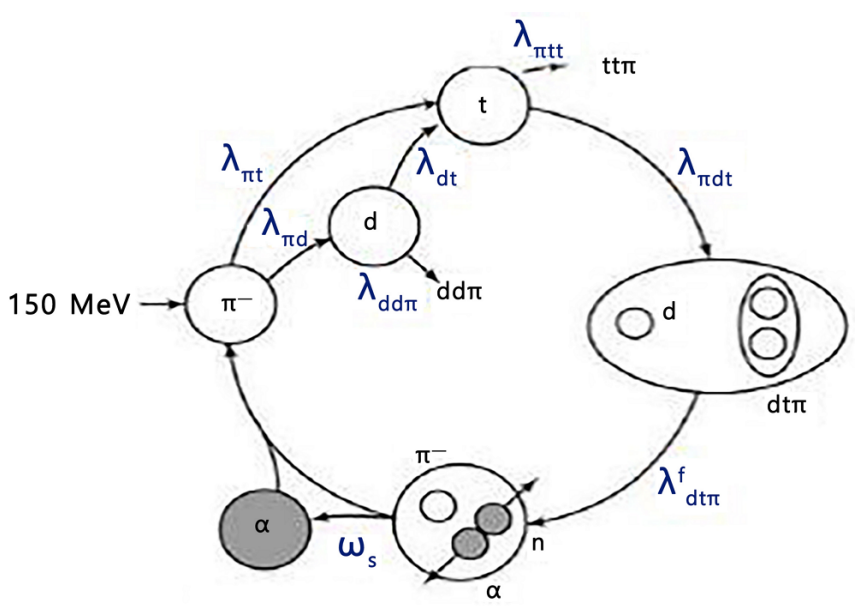

Figure 5. Pion catalytic cycle

Dynamical equations governing the particle density in the fusion system of deuterium-tritium in comprehensive network of fusion cycle, (Fig. (6)), by Pion catalyst that controls the number of all nuclei, atoms and molecules containing Pion, including a set of nonlinear coupled equations depending on time, which are provided as follows:

$$
\begin{aligned}
& \frac{\mathrm{dN}_{\pi}(\mathrm{t})}{\mathrm{dt}}=\mathrm{S}_{\pi}-\left(\lambda_{0}+\lambda_{0} \phi\right) \mathrm{N}_{\pi}(\mathrm{t})+\mathrm{P}_{1}\left(1-\omega_{\mathrm{ddt}}\right) \lambda_{\pi \mathrm{dd}}^{\mathrm{f}} \mathrm{N}_{\pi \mathrm{dd}}(\mathrm{t})+\mathrm{P}_{2}\left(1-\omega_{\mathrm{ddh}}\right) \lambda_{\pi d d}^{\mathrm{f}} \mathrm{N}_{\pi \mathrm{dd}}(\mathrm{t})+\left(1-\omega_{\mathrm{dt}}\right) \lambda^{\mathrm{f}}{ }_{\pi \mathrm{dt}} \mathrm{N}_{\pi \mathrm{dt}}(\mathrm{t})+\left(1-\omega_{\mathrm{tt}}\right) \lambda_{\pi \mathrm{tt}} \mathrm{f} \mathrm{N}_{\pi \mathrm{tt}}(\mathrm{t}) \\
& \frac{\mathrm{dN}_{\pi \mathrm{d}}(\mathrm{t})}{\mathrm{dt}}=-\left(\lambda_{0}+\mathrm{C}_{\mathrm{d}} \phi \lambda_{\pi \mathrm{dd}}+\phi \mathrm{c}_{\mathrm{t}} \lambda_{\pi \mathrm{dt}}\right) \mathrm{N}_{\pi \mathrm{d}}+\phi \mathrm{c}_{\mathrm{d}} \lambda_{\mathrm{a}} \mathrm{N}_{\pi^{(\mathrm{t})}} \\
& \frac{d N_{\pi t}(t)}{d t}=-\left(\lambda_{0}+\phi c_{t} \lambda_{\pi t t}+\phi c_{d} \lambda_{\pi d t}\right) N_{\pi t}(t)+\lambda_{a} \phi c_{t} N_{\pi}^{(t)}+\lambda_{d t} C_{t} \phi N_{\pi d}(t) \\
& \frac{\mathrm{dN}_{\pi \mathrm{dd}}(\mathrm{t})}{\mathrm{dt}}=-\left(\lambda_{0}+\lambda_{\pi \mathrm{dd}}^{\mathrm{f}}\right) \mathrm{N}_{\pi \mathrm{dd}}(\mathrm{t})+\phi \mathrm{c}_{\mathrm{d}} \lambda_{\pi \mathrm{dd}} \mathrm{N}_{\pi \mathrm{d}^{(\mathrm{t})}} \\
& \frac{\mathrm{dN}_{\pi d t}(\mathrm{t})}{\mathrm{dt}}=-\left(\lambda_{0}+\lambda_{\pi d t}^{\mathrm{f}}\right) \mathrm{N}_{\pi d t}(\mathrm{t})+\phi \mathrm{c}_{\mathrm{d}} \lambda_{\pi d t} \mathrm{~N}_{\pi \mathrm{t}}(\mathrm{t}) \\
& \frac{\mathrm{dN}_{\pi \mathrm{tt}}(\mathrm{t})}{\mathrm{dt}}=-\left(\lambda_{0}+\lambda_{\pi \mathrm{tt}}^{\mathrm{f}}\right) \mathrm{N}_{\pi \mathrm{tt}}(\mathrm{t})+\mathrm{C}_{\mathrm{t}} \phi \lambda_{\pi \mathrm{tt}} \mathrm{N}_{\pi \mathrm{t}}(\mathrm{t})
\end{aligned}
$$

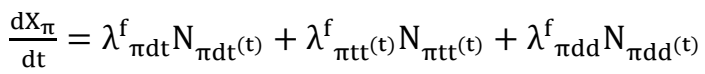




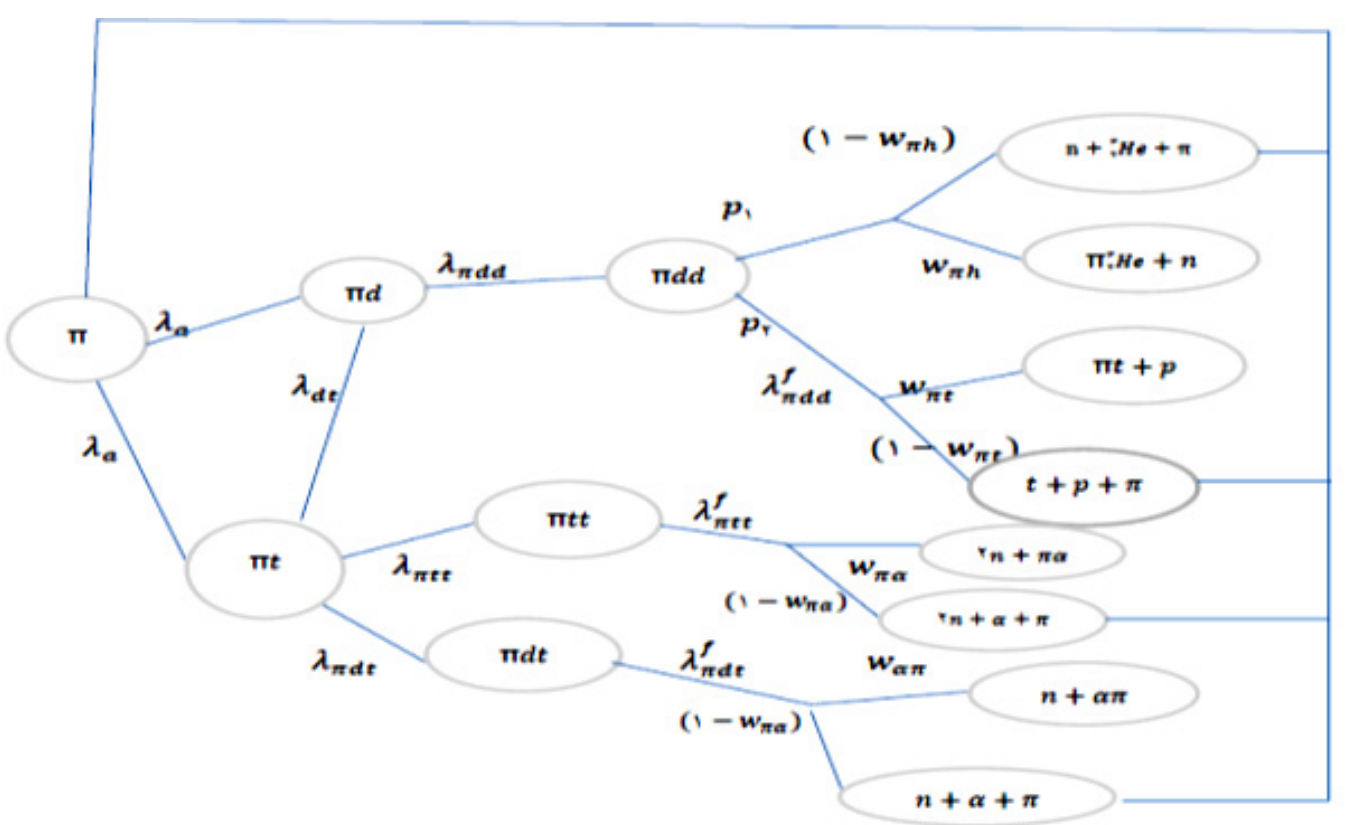

Figure 6. The Pion catalytic cycle in $\mathrm{D} / \mathrm{T}$ media

Also, the effective sticking coefficient for the proposed system is calculated as follows:

$$
\omega^{\text {eff }}=\frac{\lambda_{\pi \mathrm{dt}} \Phi \mathrm{C}_{\mathrm{d}} \omega_{\mathrm{dt}}+\lambda_{\pi \mathrm{tt}} \Phi \mathrm{C}_{\mathrm{t}} \omega_{\mathrm{tt}}}{\lambda_{0}+\lambda_{\pi \mathrm{tt}} \Phi \mathrm{C}_{\mathrm{d}}+\lambda_{\pi \mathrm{tt}} \Phi \mathrm{C}_{\mathrm{t}}}+\frac{\lambda_{\pi \mathrm{dd}} \mathrm{C}_{\mathrm{d}} \Phi\left(\mathrm{w}_{\mathrm{dd}-\mathrm{t}} \mathrm{p}_{2}+\mathrm{w}_{\mathrm{dd}-\mathrm{h}} \mathrm{p}_{1}\right)}{\lambda_{0}+\lambda_{\pi \mathrm{dd}} \Phi \mathrm{C}_{\mathrm{d}}+\lambda_{\mathrm{dt}} \Phi \mathrm{C}_{\mathrm{t}}}
$$

In writing equations (6) to (13) it should be noted that the positive statements show the atom production rate or Pionic molecule and negative sentences represent the loss of atoms or Pion molecules. $\mathrm{N}_{\pi \mathrm{ij}}, \mathrm{N}_{\pi \mathrm{i}}, \mathrm{N}_{\pi}$ are Pionic molecules density, Pionic atoms density, Pion density. Also, $\lambda_{()}$is the formation rate, $\lambda_{()}^{f}$ fusion rate, and $\Phi$ is the relative density of fuel, $C_{t}, C_{d}$ are relative density of tritium and deuterium. $\omega_{()}$and $\chi_{\pi}$ are sticking coefficient and the Pion cycle coefficient respectively. Pion cycle coefficient is the number of cycles that Pion catalyzes during fusion period and is calculated as follows.

$$
\chi_{\pi i j}=\frac{\int_{0}^{\tau} \pi \lambda_{\pi i j}^{f} N_{\pi i j}(t) d t}{\int_{0}^{\tau_{\pi}} N_{\pi}(t) d t}
$$

where $\mathrm{N}_{\pi \mathrm{ij}}$ is the molecular density of Pion, $\mathrm{i}$ and $\mathrm{j}$ isotopes of hydrogen atom and $\tau_{\pi}$ is Pion lifetime. Thus, the total Pion cycling coefficient for the $\mathrm{D} / \mathrm{T}$ media is equivalent to:

$$
\chi_{\text {total }}=\chi_{\pi \mathrm{dt}}+\chi_{\pi \mathrm{tt}}+\chi_{\pi \mathrm{dd}}
$$

The cycling rate, $\left(\lambda_{C}\right)$, represents the time of a Pion cycle which is calculated as follows:

$$
\lambda_{\mathrm{C}}=\frac{\lambda_{\circ}}{\Phi}\left(\frac{\chi_{\pi}}{1-\chi_{\pi} \mathrm{w}^{\mathrm{eff}}}\right)
$$

In order to examine the possibility of Pion catalyzed cycle in fast ignition, the dynamical equations governing the cycle (equations 6-14) have been resolved by using Monte Carlo method, and Lsod computer code in optimum physical conditions, (densities of $C_{t}=0 / 4, C_{d}=0 / 6$, and $\Phi=$ 1 LHD ) with the following initial conditions;

$$
\mathrm{N}_{\pi}(0)=1 ! \mathrm{N}_{\pi \mathrm{i}}(0)=\mathrm{N}_{\pi \mathrm{ij}}(0)=\chi_{\pi}(0)=0
$$

\section{Discussions and Conclusions}

By solving coupled dynamical equations (equations 6-14) governing the system in Physical improvement conditions, as shown in Fig. (7), it can be seen that the relative density of Pions reduces to its minimum amount after some time of about 40 (f sec). This means that $\pi^{-}$, sticks to D/T plasma ions and makes up Pionic atoms ( $\pi t$ and $\pi \mathrm{d}$ ) after a time of about 40 (f sec).

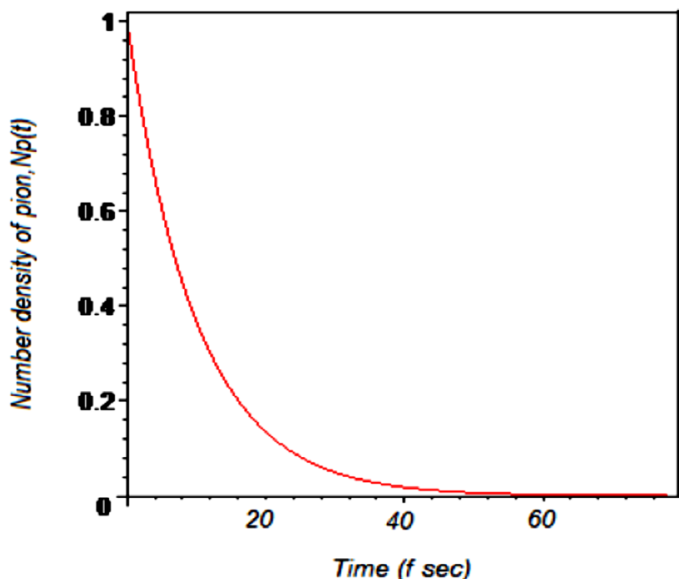

Figure 7. Relative density of Pion as function time

The number density of $\pi t$ atoms with respect to time is shown in Fig. 8. It can be seen that the relative density of $\pi t$ atom, reaches its maximum value after a time of about 1 (n $\mathrm{sec}$ ), and, after about 10 (n sec), $\pi \mathrm{t}$ atoms decay or forms $\pi \mathrm{tt}$ and $\pi \mathrm{dt}$ molecules. 


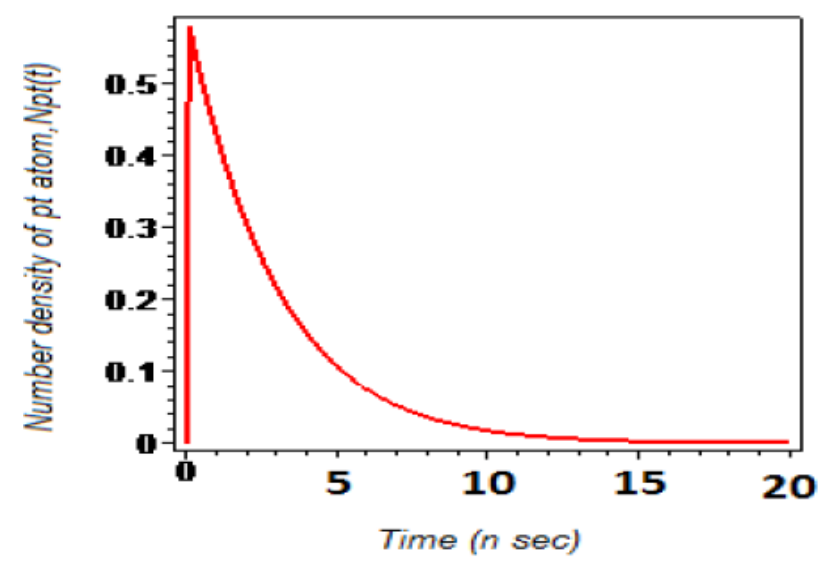

Figure 8. Relative number density of Pionic atoms( $\boldsymbol{\pi} \mathbf{t})$ as function time

According to Fig. (9), it can be observed that the number of $\pi \mathrm{dt}$ molecules, reduces to its minimum amount after some time of about 10 ( $\mathrm{n} \mathrm{sec}$ ). This means that the $\pi \mathrm{dt}$ molecules, destroyed by doing fusion process or Pion decay after about 10 (n sec).

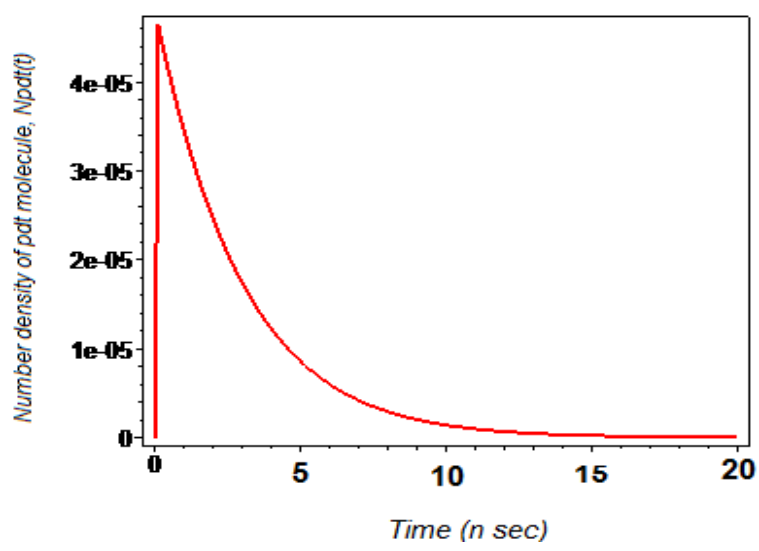

Figure 9. Number of $\pi \mathbf{d t}$ molecules as function time

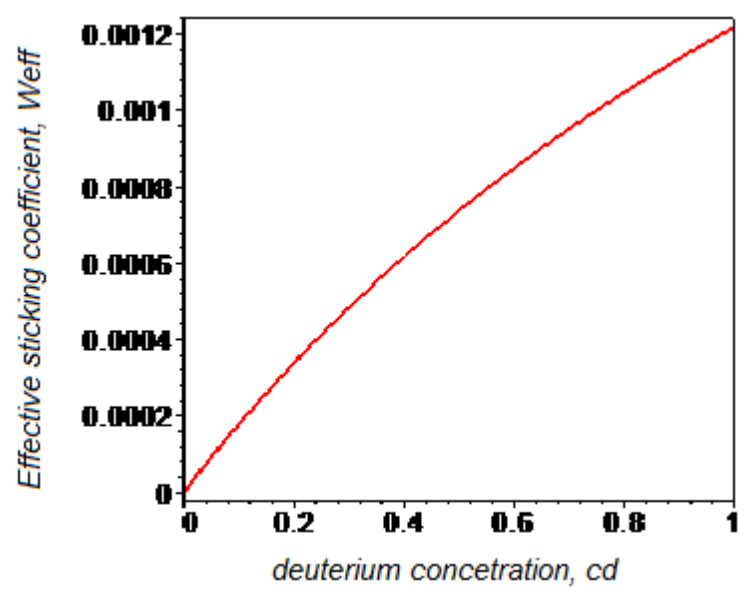

Figure 10. Effective sticking coefficient as function deuterium density

In order to calculate the Pion cycling coefficient, it is necessary that the effective sticking coefficient is calculated based on deuterium density (Fig. 10). It can be seen that the effective sticking coefficient increases with the increase of deuterium density and increases to its maximum.

By calculating pion cycling coefficient, it can be seen that, Pion cycling coefficient increases to to its maximum after some time of about 15 (n sec) ( Fig. 11).

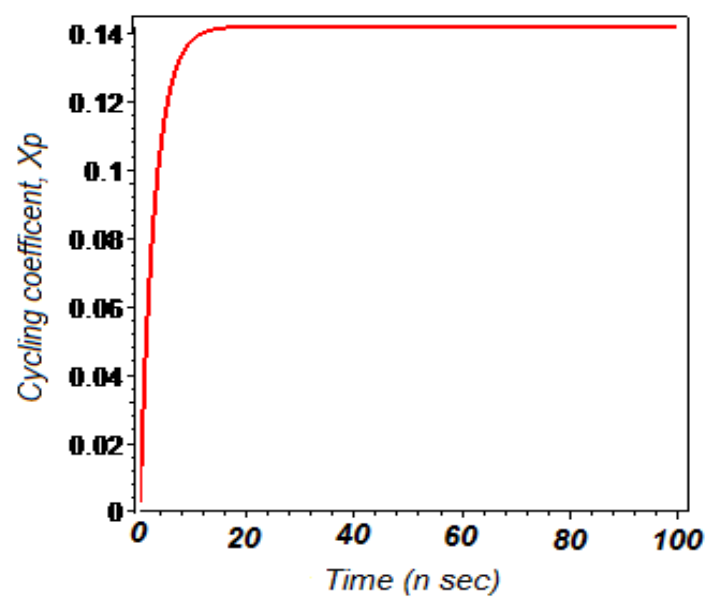

Figure 11. Pion cycling coefficient as function time

By examining and analyzing the obtained results, it can be seen that according to presence of Pion production conditions in the fast ignition process, in order to provide catalyzed fusion cycle due to the short life of Pion particles, the number of cycles that Pion catalyzes in its lifetime, is not enough to produce optimization energy. So, Pion cannot play the role of catalyst in order to produce optimization energy.

\section{REFERENCES}

[1] Chandrasekhar .S. (1961)."The stability of superposed fluids:The Rayleigh-Taylar instability, in Hydrodynamic and Hydromagnetic stability".Clarendon Press:Oxford

[2] Tatarakis. M. (2003), "Propagation Instabilties of High Intensity Laser Produced Electron Beams". Phys. Rev. lett, 90 .

[3] M. Storm.(2009)."Fast-Electron Source Characterization and Transport in High-Intensity Laser- Solid Interaction and the Role of Resistive Magnetic Field".New York: University of Rochester.

[4] Kodama.R, et al.(2002)."Nuclear fusion-fast heating scalable to laser fusion ignition".Nat.418 .933-934.

[5] Andrei Maximov, et al.(2008)"Optimizing Electron-Positron Pair Production on Kj-Class High -Intensity Lasers for the Purpose of Pair-Plasma Creation ".LLE Review. Vol.116

[6] C.Deutsch , J,-p.Didelez.(2011)."Inertial confinement fusion fast ignition with ultra-relativistic electron beams".vol.29.3944.

[7] Myatt.J ,et al. (2009)."Optimizing electron-position pair production on kilojoule-class high intensit laser for the purpose of pair-plasma creation."Phys.Rev .E. Vol 